\title{
Shape Deformation in the Brainstem of Medication-Naïve Female Patients with Major Depressive Disorder
}

\author{
Kwan Woo Choi ${ }^{1 *}$, Soonwook Kwon ${ }^{2 \star}$, Sung-Bom Pyun ${ }^{3,4}$, and Woo-Suk Tae ${ }^{4 凶}$ \\ ${ }^{1}$ Department of Psychiatry, Korea University Anam Hospital, Korea University College of Medicine, Seoul, Republic of Korea \\ ${ }^{2}$ Department of Anatomy, School of Medicine, Catholic University of Daegu, Daegu, Republic of Korea \\ ${ }^{3}$ Department of Physical Medicine and Rehabilitation, Korea University College of Medicine, Seoul, Republic of Korea \\ ${ }^{4}$ Brain Convergence Research Center, Korea University, Seoul, Republic of Korea
}

Objective Although neuroimaging studies have shown volumetric reductions, such as the anterior cingulate, prefrontal cortices, and hippocampus in patients with major depressive disorder (MDD), few studies have investigated the volume of or shape alterations in the subcortical regions and the brainstem. We hypothesized that medication-naïve female adult patients with MDD might present with shape and volume alterations in the subcortical regions, including the brainstem, compared to healthy controls (HCs).

Methods A total of 20 medication-naïve female patients with MDD and 21 age-matched female HCs, underwent 3D T1-weighted structural magnetic resonance scanning. We analyzed the volumes of each subcortical region and each brainstem region, including the midbrain, pons, and medulla oblongata. We also performed surface-based vertex analyses on the subcortical areas and brainstem.

Results Female patients with MDD showed non-significant volumetric differences in the subcortical regions, whole brainstem, and each brainstem region compared to the HCs. However, in the surface-based vertex analyses, significant shape contractions were observed in both cerebellar peduncles located on the lateral wall of the posterior brainstem [threshold-free cluster enhancement, corrected for family-wise error (FWE) at $\mathrm{p}<0.05$ ] in patients with MDD.

Conclusion We revealed shape alterations in the posterior brainstem in female patients with MDD.

Psychiatry Investig 2020;17(5):465-474

Key Words Major depressive disorder, Brainstem, Subcortical regions, Magnetic resonance imaging, Female depression.

\section{INTRODUCTION}

MDD is a complex disorder that affects emotional, cognitive processes, and quality of life throughout a patient's life. ${ }^{1-3}$ Over 300 million people suffer from major depressive disorder (MDD), which is approximately $4.4 \%$ of the world population, according to the World Health Organization (WHO). ${ }^{4}$ Neurobiological studies on MDD have suggested that structural and functional properties in the brain are the result of both environmental and genetic factors. ${ }^{5}$ To understand the etiology

Received: January 22, 2020 Revised: March 5, 2020

Accepted: March 9, 2020

$\triangle$ Correspondence: Woo-Suk Tae, $\mathrm{PhD}$

Brain Convergence Research Center, Korea University, 73 Goryedae-ro, Seongbuk-gu, Seoul 02841, Republic of Korea

Tel: +82-2-2286-1055, Fax: +82-50-4202-4629, E-mail: wstae@korea.ac.kr

*These authors contributed equally to this work.

(c) This is an Open Access article distributed under the terms of the Creative Commons Attribution Non-Commercial License (https://creativecommons.org/licenses/bync/4.0) which permits unrestricted non-commercial use, distribution, and reproduction in any medium, provided the original work is properly cited. of $\mathrm{MDD}$, numerous magnetic resonance imaging (MRI) research has attempted to investigate the neurobiological substrates of MDD and the results have supported the hypothetical pathophysiological models of MDD. ${ }^{6-9}$ Voxel-based morphometry (VBM), a fully automated method analyzing morphological MRI data, has been widely utilized to detect subtle changes in the brain structures of MDD patients. ${ }^{10}$ Recent VBM studies reported volumetric reductions in the bilateral anterior cingulate cortex (ACC), the right middle and inferior frontal gyrus, the orbitofrontal cortex (OFC), the parahippocampal gyrus, and the hippocampus. ${ }^{11-13}$ Other VBM studies have reported that subcortical structural changes and their connection with cortical regions were associated with dysfunctions in emotion processing in patients with MDD., ${ }^{2,14-16}$ However, except for the hippocampus, only a few studies have investigated volume or shape alterations in the subcortical regions, including the brainstem.

The brainstem consists of the midbrain, pons, and medulla oblongata. In the brainstem, several nuclei, such as the ventral 
tegmental area (VTA), raphe nucleus, and locus coeruleus, are associated with important monoaminergic neurotransmitters (dopamine, serotonin, and norepinephrine) in MDD. ${ }^{17,18}$ Previous transcranial sonographic (TCS) studies have reported that patients with MDD had decreased echogenicity of the brainstem raphe. ${ }^{19-21}$ Our previous study using VBM also reported a reduction in gray matter concentration in the region of the midbrain encompassing the dorsal raphe nucleus. ${ }^{22}$ However, there are conflicting study results regarding structural alterations in the brainstem of patients with MDD. SorianoMas et al. ${ }^{23}$ reported a significant increase in white matter volume within the upper brainstem tegmentum in patients with MDD. Qi et al. ${ }^{24}$ also reported that depressive patients with anxiety showed increased midbrain volume compared to HCs. More recently, our previous study similarly reported that patients with MDD had significantly greater midbrain volume compared to HCs. ${ }^{25}$ However, the above-mentioned studies only investigated volumetric differences and did not examine the effect of antidepressant treatment.

Although VBM has been widely used to identify regional brain volume changes, 10 it has been criticized for depending heavily on the accuracy and precision of image registration methods, which could result in false-positive results. ${ }^{26}$ Surface-based vertex analysis (SVA) was introduced for more efficient calculations of volume, as well as cortical thickness and surface area. ${ }^{27}$ SVA affords the quantitative measurement of brain structures by comparing the relative distances between corresponding surfaces and permits the examination of shape differences across the subcortical structures. ${ }^{27}$ The SVA analysis of subcortical structures in MDD patients demonstrates which sub-regions contribute to the overall volumetric change due to psychiatric disorders, such as MDD, and can help delineate the sub-regions contributing to symptomatic changes. ${ }^{28}$ To our knowledge, only a few studies have investigated and compared the subcortical shape alterations, especially brainstems in MDD patients and HCs.

For these reasons, we hypothesized that female adult patients with MDD might present with shape alterations and volume reductions in the brainstem compared to adult, female HCs in the present study. Since the use of antidepressants can affect volumetric changes and shape alterations in the MDD patients, ${ }^{29-31}$ we specifically hypothesized that there would be significant shape alterations and volume reductions in the brainstem in drug-naïve female MDD patients compared to HCs.

\section{MATERIALS AND METHODS}

\section{Participants}

A total of 20 female patients diagnosed with MDD were recruited from the outpatient psychiatric clinic of Kangwon
National University Hospital. We included female adults diagnosed with MDD aged 18-65 years. All MDD subjects were medication-naïve. The diagnosis of MDD was made by a boardcertified psychiatrist based on the Diagnostic and Statistical Manual of Mental Disorders, 4th Edition, Text Revision (DSMIV-TR) criteria using the Structured Clinical Interview for DSM-IV-TR Axis I Disorders (SCID-I). ${ }^{32}$ The concordance of the diagnoses for MDD was 0.95 . The exclusion criteria were 1) a presumptive primary comorbid diagnosis of any other major psychiatric illness (based on DSM-IV TR criteria) in Axis I or Axis II within the last six months, 2) MDD with psychotic features, 3) serious or unstable medical illness, 4) primary neurological illness, such as cerebrovascular disease, Parkinson's disease, or epilepsy, and 5) any contraindication for MRI. The duration of the major depressive episodes was evaluated using a life-chart methodology in the interview. Twenty-one female HCs aged 18-65 years without a history of psychiatric illness were recruited from the community to serve as the control group. Two board-certified psychiatrists independently evaluated the HCs with full psychiatric assessments to detect a present or past history of any Axis I or Axis II diagnoses. The same exclusion criteria used for the MDD patients were used for the HC group. All participants in both groups were right-handed, as assessed by the Edinburgh Handedness Test. ${ }^{33}$ The severity of the depressive symptoms in the participants in both groups was evaluated on the same day as the MRI scans using the 17-item Hamilton Depression Rating Scale (HAM-D). ${ }^{34,35}$ The details of the participants are described in Table 1 . The study protocol was approved by the Institutional Review Board of the Kangwon National University Hospital and all methods in this study were carried out in accordance with the approved guidelines and the Declaration of Helsinki. All subjects provided written informed consent to participate in the study after a full explanation and understanding of the study according to the Declaration of Helsinki.

\section{MRI acquisition}

All participants were scanned with a 1.5-T MRI scanner (Gyroscan ACS-NT; Philips Medical Systems, Best, the Netherlands). A coronal 3D T1-weighted turbo field echo MRI was obtained with the following scanning variables: $1.3 \mathrm{~mm}$ thickness; no gap; 160 slices; repetition time/echo time, 10/4.3 msec; number of signal averages, 1 ; matrix, $256 \times 256 \mathrm{~mm}$; field of view, $22 \times 22 \mathrm{~cm}$; and flip angle, $8^{\circ}$. To improve the signal-tonoise ratio, no sensitivity encoding (SENSE) acceleration factor was applied ${ }^{36}$ and so, the total scanning time for a T1 MRI was relatively long (10 $\mathrm{min} 13 \mathrm{sec}$.). Coronal slices were obtained perpendicular to the long axis of the anterior commissure to the posterior commissure in the midsagittal plane. The final voxel size was $0.86 \times 0.86 \times 1.30 \mathrm{~mm}(\mathrm{x} \times \mathrm{y} \times \mathrm{z})$. 
KW Choi et al.

Table 1. Clinical characteristics at baseline $(\mathrm{N}=41)$

\begin{tabular}{|c|c|c|c|c|}
\hline & $\operatorname{MDD}(\mathrm{N}=20)$ & $\mathrm{HC}(\mathrm{N}=21)$ & t or $\chi^{2}$ & $\mathrm{p}$ value \\
\hline Age (mean, SD) & $42.25 \pm 13.65$ & $42.33 \pm 10.24$ & 0.031 & 0.982 \\
\hline Education level & & & & $0.003^{*}$ \\
\hline Elementary and middle school & 11 & 2 & 11.797 & \\
\hline High school or college/university & 7 & 9 & & \\
\hline Above graduate school & 2 & 10 & & \\
\hline Family history of MDD (yes/no) & $5 / 15$ & $0 / 30$ & 5.979 & $0.021^{*}$ \\
\hline HAM-D (mean, SD) & $21.72 \pm 8.67$ & $0.95 \pm 1.23$ & 10.074 & $<0.0001^{*}$ \\
\hline Onset of MDE (age, years, mean, SD) & $31.68 \pm 13.10$ & - & & \\
\hline Duration of MDE (months, mean, SD) & $114.22 \pm 135.45$ & - & & \\
\hline Number of MDE (mean, SD) & $7.12 \pm 9.12$ & - & & \\
\hline Number of suicide attempts (mean, SD) & $0.56 \pm 0.71$ & - & & \\
\hline
\end{tabular}

${ }^{*} \mathrm{p}<0.05$. MDD: major depressive disorder, HC: healthy controls, MDE: major depressive episode, HAM-D: Hamilton depression rating scale

\section{Automated segmentation}

Automated segmentation of the subcortical nuclei (caudate nucleus, putamen, nucleus accumbens, thalamus, pallidum, hippocampus, amygdala, and brainstem) and surface-based vertex analyses were performed using the FMRIB Software Library (FSL) version 5.0.9 (http://fsl.fmrib.ox.ac.uk/fsl). Automatic segmentations of the subcortical nuclei were processed using the FIRST algorithm, ${ }^{27}$ which is a fully automated segmentation software included in the FSL package. The FIRST segmentation algorithm is based on appearance models and shapes generated from 336 manually labeled reference images and this is combined with a Bayesian probability using a Gaussian assumptions approach. The segmentation processes involved a two-step affine transformation to an MNI (Montreal Neurologic Institute) 152 standard space with a $1 \mathrm{~mm}$ isotropic resolution. The first process was a spatial registration to the MNI 152 template with the standard 12 degrees of freedom. The second process was 12 degrees of freedom (DOF) spatial registration using an MNI152 subcortical mask. The accuracy of the segmentation was visually checked for all subjects. The total intracranial cavity volume (TICV) was manually measured $^{37}$ to normalize the habitual brain size.

\section{Surface-based vertex analysis}

The surface-based vertex shape analyses were processed using the FIRST tool. ${ }^{27}$ This analysis aimed to yield statistical group differences on a per-vertex basis by a multivariate general linear model. Surface meshes of segmented subcortical nuclei represent the volumetric information of each nucleus and are composed of parameterized deformable surfaces. These shape analyses were processed in the native space and the vertex locations from each nucleus were projected onto the surface of each average shape for all subjects.

Scalar distances from the mean surface were processed us- ing the randomise command, which tests univariate permutations at each vertex point. ${ }^{38}$ The multiple-comparison problem was corrected at the cluster level using threshold-free cluster enhancement (TFCE) ${ }^{39}$ with a family-wise error (FWE) rate of $\mathrm{p}<0.05$. The between-group comparisons were independently tested using analysis of covariance (ANCOVA) and confounders of age, TICV, and the duration of education with 5,000 permutations. The statistical group differences were visualized with the statistical masks of each region, which showed significant differences.

\section{Brainstem sub-regional volumetry}

Four sub-brainstem structures (midbrain, pons, medulla oblongata, and superior cerebellar peduncle) were automatically labeled and each volume was extracted from the individual T1 MRIs of all participants using the image processing pipeline for brainstem in FreeSurfer 6.0 (Massachusetts General Hospital, Boston, MA, USA, http://surfer.nmr.mgh.harvard. edu). The subregional brainstem segmentation of Freesurfer uses a probabilistic atlas of the brainstem and its neighboring brain structures. This method allows for the subregional segmentation of the brainstem using a robust and accurate Bayesian algorithm. The technical details of this procedure have been previously described..$^{40}$ In brief, image processing of the brain MRIs was performed using the brainstem pipeline implemented in Freesurfer. The preprocessing procedures included skull stripping, bias field correction, ${ }^{41}$ automated spatial transformation to the standard space of each subject's brain, ${ }^{42,43}$ intensity normalization, and the segmentation of subcortical structures. ${ }^{42}$ After the preprocessing procedures, the preprocessed brain images were fed into the fully automatic segmentation algorithm implemented in FreeSurfer and the labels and volumes of the midbrain, pons, medulla oblongata, and superior cerebellar peduncle were estimated (Figure 1). ${ }^{40}$ 


\section{Statistical analyses}

The mean differences in age, TICV, clinical characteristics, and demographic variables of the MDD patients and normal controls were analyzed using t-tests and the gender distribution was compared using chi-squared tests. The volumes of the bilateral caudate nuclei, putamina, nuclei accumbens, thalami, pallidum, hippocampi, amygdalae, and brainstem calculated from the FSL software were tested using ANCOVA with individual volumes as dependent variables, groups as an independent variable, and age, TICV, and duration of education, as covariates. The volumes of the whole brainstem and its subregions, including the medulla, pons, supracerebellar peduncle (SCP), and midbrain, were tested using ANCOVA with individual volumes as dependent variables, groups as an independent variable, and age, TICV, and duration of education as covariates.

All statistical analyses were performed using SPSS version 24.0 (IBM Corp., Armonk, NY, USA). All volumetric analysis statistics were corrected for multiple comparison problems using the false discovery rate (FDR) method by Benjamini and Hochberg. ${ }^{44}$

\section{RESULTS}

\section{Characteristics of participants}

The characteristics of the participants, including age, education level, family history of MDD, family history of depression, HAM-D, age at disease onset, the duration of illness, and the number of depressive episodes and suicide attempts are presented in Table 1. In the chi-squared test, patients in the MDD group were significantly more likely to have a family history of depression $(\mathrm{p}=0.021)$ and lower education level $(\mathrm{p}=$ 0.003) than the HCs, as shown in Table 1.

\section{Surface-based shape analysis}

In the surface-based vertex analyses, significant shape contractions were observed in both cerebellar peduncles located on the lateral wall of the posterior brainstem (TFCE corrected $\mathrm{p}<0.05)$. However, there were no local shape deformations in the bilateral caudate nuclei, putamina, nuclei accumbens, thalami, pallidum, hippocampi, and amygdalae (Figures 2 and 3).

\section{Volumetric analysis}

In the subcortical areas, the volume changes in the putamina of MDD patients were significant at the level of uncorrected $\mathrm{p}$-values (right, $\mathrm{p}=0.009$; left, $\mathrm{p}=0.021$ ), but were not significant after FDR correction. TICV was not different between the two groups (Table 2). In the whole brainstem and its subregion areas, there were no significant differences between MDD and HCs (Table 3).

\section{DISCUSSION}

To our knowledge, this was the first study to investigate shape alterations in the brainstem of medication-naïve middle-aged female MDD patients. In the present study, we revealed that patients with MDD had significant shape contractions in the posterior brainstem compared to female HCs. Since we only recruited and analyzed patients with MDD who were medication-naïve and female, we could rule out the effects of medication and sex. However, there were no significant shape

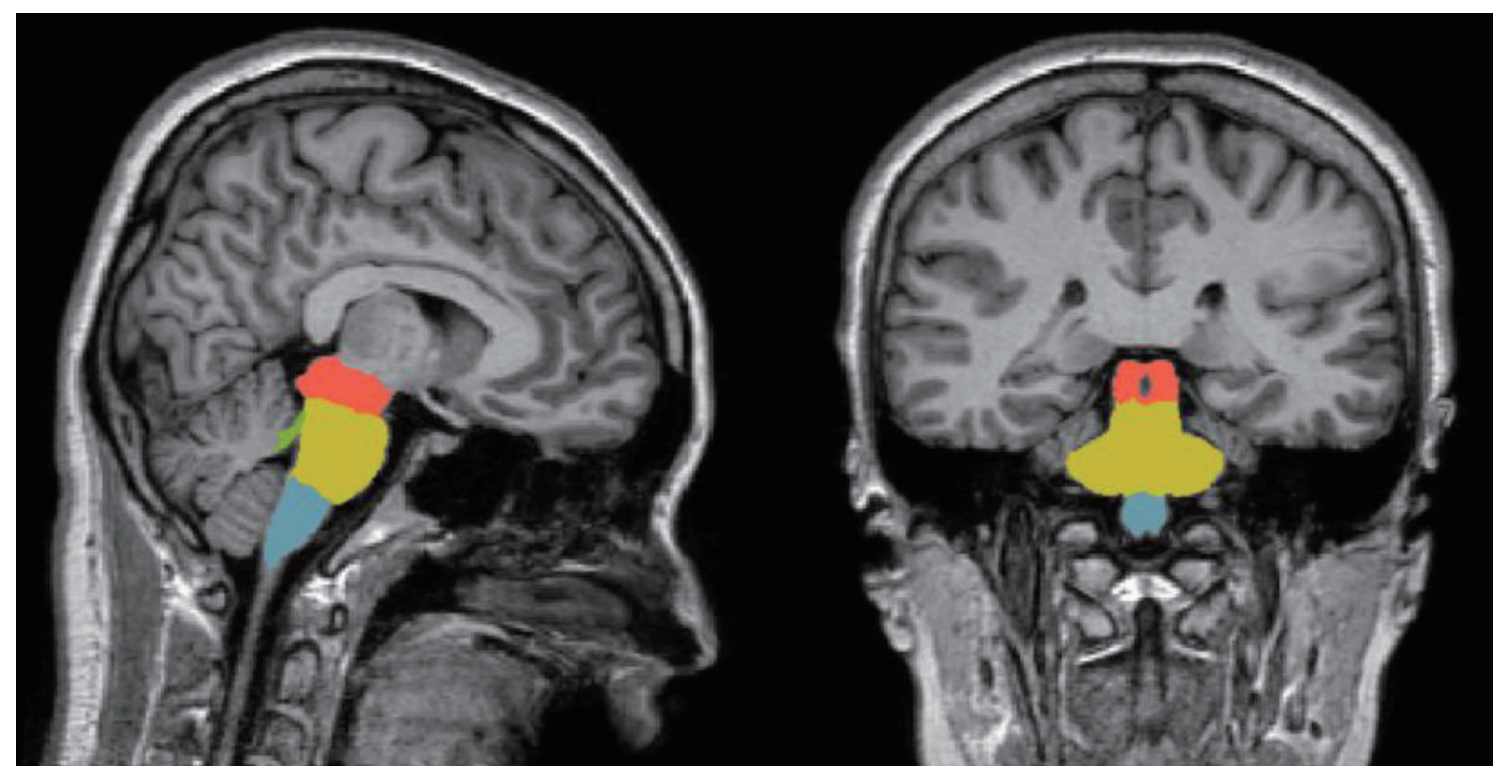

Figure 1. Automated brainstem parcellation. Brain 3D T1 MRI was automatically labeled by the brainstem pipeline of FreeSurfer. The midbrain (orange), pons (yellow), superior cerebellar peduncle (green), and medulla oblongata (cyan) are displayed in the sagittal (left) and coronal (right) views. 


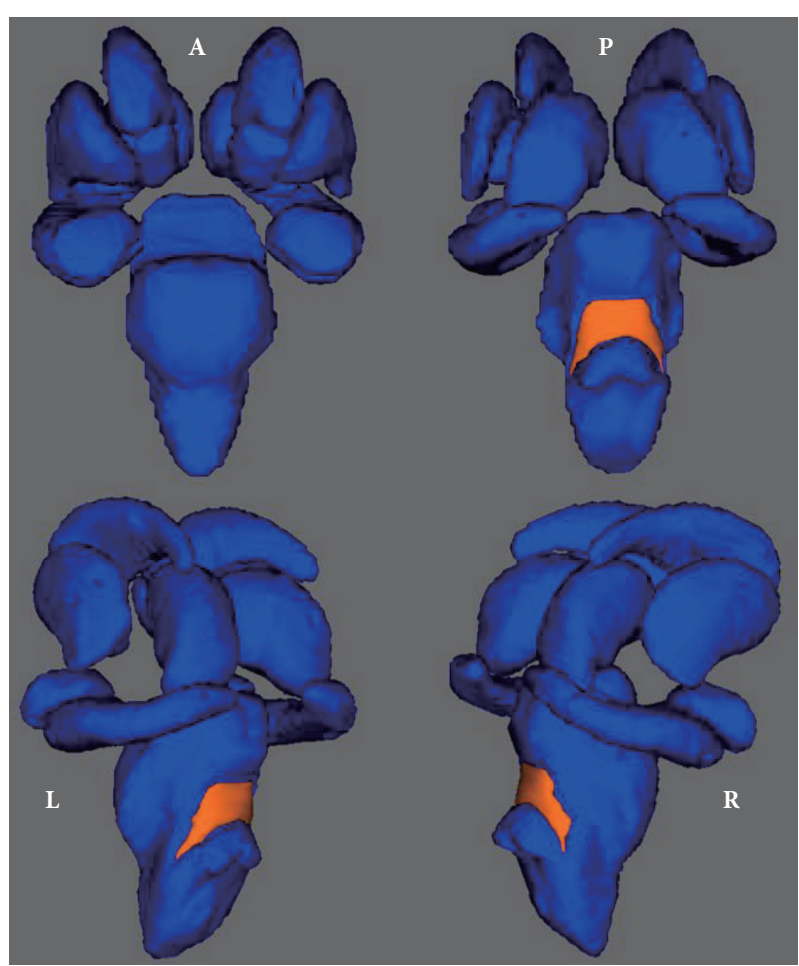

Figure 2. The results of surface-based vertex analyses of all subcortical nuclei. The bilateral posterior brainstem of a major depressive disorder patient showing regionally contracted shape deformation at the corrected $p<0.05$ significance level for threshold-free cluster enhancement. There were no shape deformations in the caudate nuclei, putamina, nuclei accumbens, thalami, pallidum, hippocampi, or amygdali.

alterations in the other subcortical regions, except for the brainstem, in the medication-naïve female patients with MDD. Also, there were no significant volumetric differences in the subcortical regions, including the brainstem and each brainstem region, between the medication-naïve female patients with $\mathrm{MDD}$ and female HCs.

The current study is consistent with previous research findings which showed significant structural or functional changes in the brainstem regions in MDD patients compared to HCs. ${ }^{19-23,25,45-47}$ Previously, TCS studies showed that patients with MDD had decreased echogenicity of the brainstem raphe. ${ }^{19-21,47}$ Furthermore, Budisic et al. ${ }^{47}$ reported that the echogenicity of the raphe nuclei in the midbrain was associated with depression severity and suicidal ideation. Using diffusor tensor imaging, Bessette et al. ${ }^{46}$ also found that adolescents with MDD showed significantly lower fractional anisotropy in the midbrain compared to HCs. One of our previous VBM studies reported a structural reduction in gray matter concentration of the midbrain encompassing the dorsal raphe nucleus. ${ }^{22}$ In contrast, several studies showed an increased volume of the brainstem in patients with MDD compared to HCs. ${ }^{23,25}$ Soriano-Mas et al. ${ }^{23}$ investigated gray and white matter volume changes in the whole brain of 70 patients of MDD and

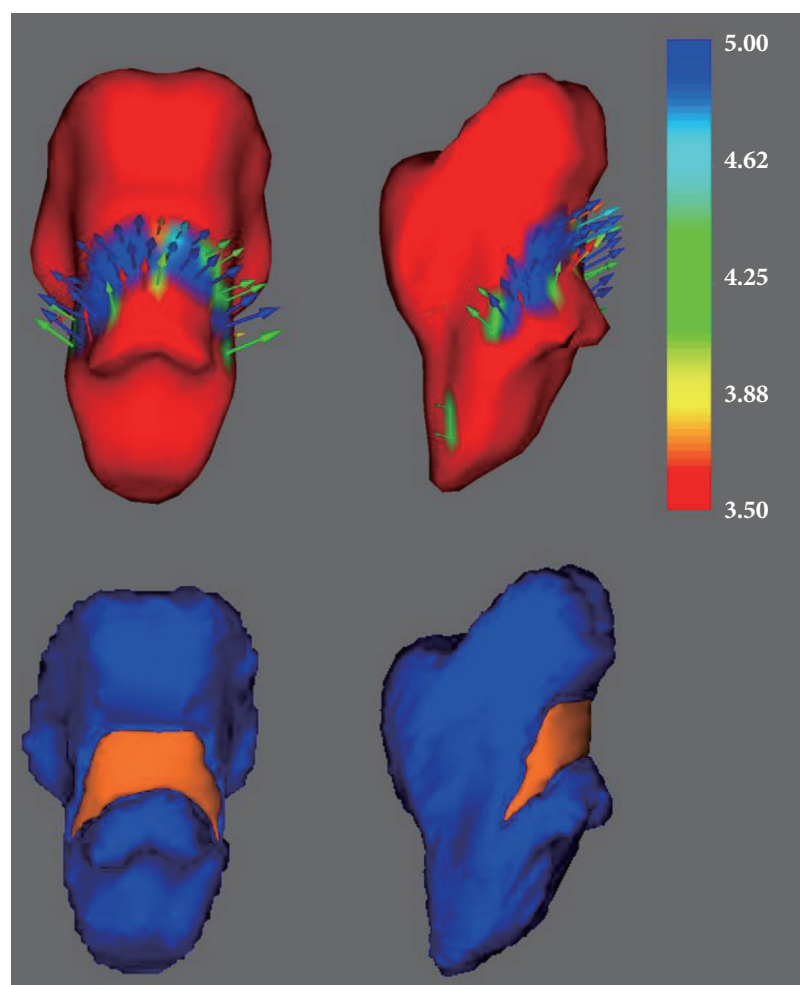

Figure 3. Shape deformations of the brainstem with vector scale. The vector arrows represent the outward deformation of the normal control group (inward deformation in MDD) compared to the MDD patient group (upper row). The corresponding surfacebased vertex analyses are in the lower row. The colored bar represents the T-values. MDD: major depressive disorder.

40 HCs. They found that patients with melancholic depression showed white matter volume increases in the upper brainstem tegmentum, at the level of the midbrain, and the rostral pons. Han et al. ${ }^{48}$ also reported that drug-naïve patients with MDD showed significantly greater midbrain volumes compared to HCs. Different from the above-mentioned two studies, ${ }^{23,25}$ our findings showed significant shape reductions in the brainstem, at the level of the posterior pons and medulla in medication-naïve female patients compared to HCs. Because of the relatively longer illness duration in our study participants than Han et al'. ${ }^{25}$ participants (114.22 vs. 50.76), the shape reduction in the brainstem might have progressed from hyperactivation of the brainstem to shrinkage or loss of function of this area. Similarly, Soriano-Mas et al..$^{23}$ also showed that patients with MDD had reduced white matter volumes in the upper brainstem tegmentum during the 7-year observation period in their longitudinal assessment.

In the present study, we found shape contraction in the posterior pons and medulla in medication-naïve female patients with MDD compared to female HCs. The brainstem performs vital roles for life, including the autonomous control of the internal organs and recent research has revealed that the brainstem is associated with emotional changes in the limbic sys- 
Table 2. The differences in subcortical volumes between normal controls and MDD patients

\begin{tabular}{|c|c|c|c|c|c|c|}
\hline Regions $\left(\mathrm{mm}^{3}, \mathrm{SD}\right)$ & & $\operatorname{MDD}(\mathrm{N}=20)$ & $\mathrm{HC}(\mathrm{N}=21)$ & $\mathrm{F}$ & $\mathrm{p}$ & FDR p \\
\hline TICV & & $1,416.7 \pm 109.52$ & $1,395.9 \pm 120.48$ & 0.421 & 0.568 & ns \\
\hline Stem & & $20.28 \pm 1.562$ & $20.90 \pm 2.060$ & 3.204 & 0.640 & ns \\
\hline Thalamus & $\mathrm{Lt}$ & $7.34 \pm 0.573$ & $7.42 \pm 0.658$ & 0.450 & 0.505 & ns \\
\hline Thalamus & Rt & $7.01 \pm 0.605$ & $7.11 \pm 0.654$ & 0.454 & 0.584 & ns \\
\hline Caudate nucleus & $\mathrm{Lt}$ & $3.07 \pm 0.282$ & $3.17 \pm 0.292$ & 1.805 & 0.635 & ns \\
\hline Caudate nucleus & Rt & $3.17 \pm 0.254$ & $3.27 \pm 0.318$ & 2.256 & 0.446 & ns \\
\hline Putamen & $\mathrm{Lt}$ & $4.85 \pm 0.474$ & $4.74 \pm 0.508$ & 0.299 & 0.021 & ns \\
\hline Putamen & Rt & $4.90 \pm 0.444$ & $4.78 \pm 0.445$ & 0.434 & 0.009 & ns \\
\hline Pallidum & $\mathrm{Lt}$ & $1.56 \pm 0.233$ & $1.67 \pm 0.241$ & 2.760 & 0.844 & ns \\
\hline Pallidum & Rt & $1.68 \pm 0.163$ & $1.70 \pm 0.194$ & 0.308 & 0.419 & ns \\
\hline Hippocamous & $\mathrm{Lt}$ & $3.71 \pm 0.346$ & $3.73 \pm 0.446$ & 0.149 & 0.485 & ns \\
\hline Hippocamous & Rt & $3.91 \pm 0.279$ & $3.83 \pm 0.455$ & 0.184 & 0.161 & ns \\
\hline Amygdala & $\mathrm{Lt}$ & $1.20 \pm 0.171$ & $1.23 \pm 0.178$ & 0.427 & 0.546 & ns \\
\hline Amygdala & Rt & $1.25 \pm 0.197$ & $1.24 \pm 0.134$ & 0.032 & 0.671 & ns \\
\hline Accumbens & $\mathrm{Lt}$ & $0.56 \pm 0.871$ & $0.61 \pm 0.824$ & 6.135 & 0.254 & ns \\
\hline Accumbens & Rt & $0.43 \pm 0.772$ & $0.46 \pm 0.071$ & 2.448 & 0.931 & ns \\
\hline
\end{tabular}

The $\mathrm{p}$ values for comparison in age and TICV were obtained by independent t-tests; The group differences in subcortical volumes were tested using analysis of covariance with age, duration of education, and TICV as covariates. The data are expressed as mean \pm standard deviation $\left(\mathrm{mm}^{3}\right)$. MDD: major depressive disorder, HC: healthy controls, FDR: false discovery rate by Benjamini and Hochberg, ns: not significant, TICV: total intracranial cavity volume

Table 3. The differences in volumes of the brainstem subregions between normal controls and MDD patients

\begin{tabular}{lccccc}
\hline \multicolumn{1}{c}{ Regions } & Normal controls & MDD & F & p & FDR p \\
\hline Medulla & $4,197.9 \pm 397.36$ & $4.134 .6 \pm 297.05$ & 0.683 & 0.414 & $\mathrm{~ns}$ \\
Pons & $13,143.4 \pm 1,530.58$ & $12,983,4 \pm 1,305.66$ & 0.341 & 0.563 & $\mathrm{~ns}$ \\
SCP & $221.2 \pm 42.58$ & $237.6 \pm 36.37$ & 1.724 & 0.197 & $\mathrm{~ns}$ \\
Midbrain & $5,440.6 \pm 444.35$ & $5,337.5 \pm 380.15$ & 2.294 & 0.138 & $\mathrm{~ns}$ \\
Whole brainstem & $23,003.0 \pm 2,266.93$ & $22,693.1 \pm 1,844.46$ & 0.652 & 0.425 & $\mathrm{~ns}$ \\
\hline
\end{tabular}

The volumetric group differences in subcortical nuclei were tested using analysis of covariance with age, duration of education, and TICV as covariates. The data are expressed as mean \pm standard deviation $\left(\mathrm{mm}^{3}\right)$. MDD: major depressive disorder, FDR: false discovery rate by Benjamini and Hochberg, ns: not significant, SCP: supracerebellar peduncle

tem. ${ }^{49}$ The brainstem is composed of numerous nuclei (neural cell bodies) and tracts (axon fibers). Several nuclei, such as the dorsal raphe nucleus and locus coeruleus, are located on the posterior surface of the brainstem. The dorsal raphe nucleus is serotonergic, connected to the prefrontal cortex and limbic system. The connection starts from the limbic system runs to the dorsal raphe nucleus. ${ }^{50}$ This implies that the brainstem can affect the limbic system, which controls emotion. These connections have been reported in animal studies and regulation of cellular processes, such as transcription and methylation, have been associated with MDD. ${ }^{51-53}$ The results of these previous studies show that a decrease in dorsal raphe nuclei could affect the limbic system, resulting in MDD or as a result of MDD. The locus coeruleus activates almost all parts of the cerebrum by noradrenergic action, which is also connected to the limbic system controlling emotion..$^{54}$
Neuroimaging studies showed gray matter reduction in various regions of the brain in patients with MDD. These findings are supported by various previous reports showing that gray matter reduction existed simultaneously in the bilateral nuclei accumbens, dorsal raphe nuclei, and cerebellum, or changes in the transporter system in these regions. ${ }^{48,55-57}$ Other neuroimaging studies directly focused on the brainstem also supported these findings. ${ }^{21,45,49}$ The locus coeruleus is composed of neurons secreting norepinephrine and is observed in the posterior pons. Our findings that the surface of the brainstem was reduced could imply atrophy of the locus coeruleus in patients with MDD. The locus coeruleus is connected to almost all cerebral regions and activates them, ${ }^{58,59}$ especially the amygdala, hippocampus, and thalamus, areas correlated with MDD ${ }^{60,61}$ Input from medullary noradrenergic nuclei is directed to the forebrain structure, which affects the functions of the 
hypothalamus. Moreover, the hypothalamic-pituitary-adrenal (HPA) axis affected by stress behavior is controlled by glucocorticoid receptors, which could be damaged by depression. Because the glucocorticoid receptors are in the locus coeruleus, a damaged locus coeruleus could reduce negative feedback to the HPA axis mediated by the glucocorticoid receptor. ${ }^{62} \mathrm{~A}$ postmortem study revealed that noradrenergic transporters in the locus coeruleus were reduced in patients with $\mathrm{MDD}^{63}$ and an MRI study also showed a reduction in the rostral and middle portions of the locus coeruleus in these patients. ${ }^{64}$ Another postmortem study reported changes in transcription and DNA methylation in the galanin receptor in the locus coeruleus, which were also reported in an animal study. ${ }^{65}$ Elevated gene expression of the glutamate receptor in the locus coeruleus was confirmed in patients with depression who committed suicide. ${ }^{66}$ These postmortem studies provide clues that a decrease in the locus coeruleus may have an influence on MDD.

Among the neural tracts, the solitary tract passing through the pons is well known for its paths, which is consistent with our results. The connection of the brainstem to the amygdala is known as the solitary connection to the limbic system. Thus, the solitary tract is an important connection between the medulla and the amygdala, which has a critical role in the regulation of the HPA axis. ${ }^{67,68}$ Because the amygdala is largely affected in MDD, damage to the tracts that connect the amygdala and other nuclei could critically influence MDD. Astrocytes are known as supporters of the neural structure and represent the largest number of glial cells. ${ }^{69}$ They are about 10 to 50 times more astrocytes than other glial cells. Astrocytes represent the largest proportion of cells in the neural system. ${ }^{70}$ Recent studies reported the expanded roles of astrocytes, not only in housekeeping, but also in synaptic strength, synaptogenesis, stability, and hippocampal neurogenesis. In addition to this, antidepressants regulate astroglia-specific proteins and intracellular signaling. ${ }^{71}$ Some postmortem studies reported that the frontolimbic area of MDD patients showed a reduced number of glial cells and alterations. ${ }^{72,73}$ Although this might not be direct proof of the reduced number of glial cells in the brainstem, an overall reduction in glial cells was reported in the whole brain, including the dorsolateral prefrontal cortex and amygdala. ${ }^{73,74}$ This suggests that a reduction in the number of astrocytes as a result of MDD could occur, not only in the cerebrum but also in the whole brain, including the brainstem. Therefore, we suggest that one of the reasons for posterior brainstem reduction seen in our study could come from glial cells, not the nuclei or tracts.

This study recruited and analyzed only female participants whose mean-age was in the forties. Sex and gender differences in MDD are well-recognized and among the most interesting and consistent findings in psychiatry. ${ }^{75,76}$ Epidemiologic re- search reported substantial sex or gender-related differences in MDD prevalence and these studies consistently showed that the incidence and prevalence of MDD in adult females were approximately two times higher than in adult males. ${ }^{77-84}$ These sex and gender differences increase at puberty and from early adulthood to late in life. Both sexes display a parallel course, with a female predominance in the prevalence of MDD in the adult age groups. ${ }^{77-84}$ Even after menopause, the female preponderance in depression continues late into life. ${ }^{85,86}$ However, although these sex and gender differences in MDD have been poorly understood, there is a paucity of research on the etiological mechanisms regarding this issue, especially investigations using brain MRI. Further studies are needed to investigate the gender effect on brain functions and structures in MDD patients.

This study had several limitations and the results should be interpreted with caution. First, a small number of subjects could cause statistically insignificant results. To overcome the small sample size, we included only female subjects to increase the sample homogeneity and the ages of the two groups were closely matched. Second, the surface analysis could not directly reveal changes in the nuclei or tracts in the brainstem. However, it is well known that the volume of prominent structures, such as the superior colliculi, is effected by neurons, especially neural cell bodies. This could mean that the opposite effect of reduced volume in the surface analysis resulted from the decreased number of neural cell bodies or tracts. Although the reason for the reduction was mainly from the cell bodies, we could not exclude the tracts, which was a limitation of the neuroimaging technique. Thus, future studies should overcome this limitation by histological analyses. Third, since we only recruited and analyzed the data from female only, we could not show sex-specific morphometric changes of brainstem in the female MDD patients compared to male MDD patients. Sex hormones could have different effects on various brain structures or functions or different responses in MDD patients. In the present data, the average age of the subjects was 42.5 years, which suggests hormonal support in the subjects and thus, male patients with MDD could exert different influences on their brainstem. We hope that further studies will include male subjects to reveal sex differences in the brain structure influenced by depression. Fourth, the education levels of the groups were different. Therefore, education level was included as an independent variable in the linear regression analysis with stepwise variable selection method to examine whether differences in educational levels affected the results seen regarding volume reduction in the subcortical nuclei. No significant association was observed between educational levels and the volume of subcortical nuclei in each group, suggesting that education level did not affect the volume or shape changes seen 
in this study.

We revealed an atrophy-like effect on the posterior brainstem in medication-naïve female patients with MDD. Because these regions have networks connecting to the limbic system or other cerebral structures, it is important to focus on the brainstem in patients with MDD. To investigate the findings of this study in greater detail, we suggest that further advanced neuroimaging studies with high-resolution MRI, specifically involving the brainstem, are performed.

\section{Acknowledgments}

This research was supported the Bio \& Medical Technology Development Program of the National Research Foundation (NRF) funded by the Ministry of Science, ICT and Future Planning (NRF-2017M3C7A1079696), and by the Research Program to Solve Social Issues of the NRF of Korea funded by the Ministry of Science and ICT (MSIT) (NRF-2017R1D1A1B03030280). This work was also supported and funded by the NRF grant funded by the Korea government (MSIT) (NRF-2020R1C1C1003394), and by Korea University (K1922891).

The NRF of Korea and Korea University had no further role in study design; collection, analysis, and interpretation of data; writing of the report; or in the decision to submit the study for publication.

\section{Conflicts of Interest}

The authors have no potential conflicts of interest to disclose.

\section{Author Contributions}

Conceptualization: Woo-Suk Tae. Data curation: Woo-Suk Tae, Kwan Woo Choi. Formal analysis: Woo-Suk Tae, Soonwook Kwon. Investigation: Soonwook Kwon, Kwan Woo Choi. Methodology: Woo-Suk Tae. Project administration: Woo-Suk Tae. Supervision: Woo-Suk Tae, Sung-Bom Pyun. Validation: Woo-Suk Tae. Visualization: Woo-Suk Tae. Writingoriginal draft: Soonwook Kwon. Writing—review \& editing: Woo-Suk Tae, Kwan Woo Choi.

\section{ORCID iDs}

$\begin{array}{ll}\text { Kwan Woo Choi } & \text { https://orcid.org/0000-0002-0854-3507 } \\ \text { Soonwook Kwon } & \text { https://orcid.org/0000-0002-3945-2894 } \\ \text { Sung-Bom Pyun } & \text { https://orcid.org/0000-0002-1933-038X } \\ \text { Woo-Suk Tae } & \text { https://orcid.org/0000-0003-0451-0713 }\end{array}$

\section{REFERENCES}

1. Papakostas GI, Petersen T, Mahal Y, Mischoulon D, Nierenberg AA, Fava M. Quality of life assessments in major depressive disorder: a review of the literature. Gen Hosp Psychiatry 2004;26:13-17.

2. Rive MM, van Rooijen G, Veltman DJ, Phillips ML, Schene AH, Ruhe HG. Neural correlates of dysfunctional emotion regulation in major depressive disorder. A systematic review of neuroimaging studies. Neurosci Biobehav Rev 2013;37:2529-2553.

3. McIntyre RS, Cha DS, Soczynska JK, Woldeyohannes HO, Gallaugher LA, Kudlow P, et al. Cognitive deficits and functional outcomes in major depressive disorder: determinants, substrates, and treatment interventions. Depress Anxiety 2013;30:515-527.

4. World Health Organization. Depression and Other Common Mental Disorders: Global Health Estimates. Geneva: World Health Organization; 2017.

5. Kupfer DJ, Frank E, Phillips ML. Major depressive disorder: new clinical, neurobiological, and treatment perspectives. Lancet 2012;379: 1045-1055.

6. Drevets WC, Price JL, Furey ML. Brain structural and functional abnormalities in mood disorders: implications for neurocircuitry models of depression. Brain Struct Funct 2008;213:93-118.

7. Marchand WR, Yurgelun-Todd D. Striatal structure and function in mood disorders: a comprehensive review. Bipolar Disord 2010;12:764785.

8. Mayberg HS. Limbic-cortical dysregulation: a proposed model of depression. J Neuropsychiatry Clin Neurosci 1997;9:471-481.

9. Pizzagalli DA. Frontocingulate dysfunction in depression: toward biomarkers of treatment response. Neuropsychopharmacology 2011;36: 183-206.

10. Ashburner J, Friston KJ. Voxel-based morphometry--the methods. Neuroimage 2000;11:805-821.

11. Bora E, Fornito A, Pantelis C, Yucel M. Gray matter abnormalities in Major Depressive Disorder: a meta-analysis of voxel based morphometry studies. J Affect Disord 2012;138:9-18.

12. Du MY, Wu QZ, Yue Q, Li J, Liao Y, Kuang WH, et al. Voxelwise metaanalysis of gray matter reduction in major depressive disorder. Prog Neuropsychopharmacol Biol Psychiatry 2012;36:11-16.

13. Lai CH. Gray matter volume in major depressive disorder: a metaanalysis of voxel-based morphometry studies. Psychiatry Res 2013; 211:37-46.

14. Koolschijn PC, van Haren NE, Lensvelt-Mulders GJ, Hulshoff Pol HE, Kahn RS. Brain volume abnormalities in major depressive disorder: a meta-analysis of magnetic resonance imaging studies. Hum Brain Mapp 2009;30:3719-3735.

15. Schmaal L, Veltman DJ, van Erp TG, Samann PG, Frodl T, Jahanshad $\mathrm{N}$, et al. Subcortical brain alterations in major depressive disorder: findings from the ENIGMA Major Depressive Disorder working group. Mol Psychiatry 2016;21:806-812.

16. Schmaal L, Hibar DP, Samann PG, Hall GB, Baune BT, Jahanshad N, et al. Cortical abnormalities in adults and adolescents with major depression based on brain scans from 20 cohorts worldwide in the ENIGMA Major Depressive Disorder Working Group. Mol Psychiatry 2017; 22:900-909.

17. D’Ardenne K, McClure SM, Nystrom LE, Cohen JD. BOLD responses reflecting dopaminergic signals in the human ventral tegmental area. Science 2008;319:1264-1267.

18. Gervais J, Rouillard C. Dorsal raphe stimulation differentially modulates dopaminergic neurons in the ventral tegmental area and substantia nigra. Synapse 2000;35:281-291.

19. Becker G, Becker T, Struck M, Lindner A, Burzer K, Retz W, et al. Reduced echogenicity of brainstem raphe specific to unipolar depression: a transcranial color-coded real-time sonography study. Biol Psychiatry 1995;38:180-184.

20. Becker G, Struck M, Bogdahn U, Becker T. Echogenicity of the brainstem raphe in patients with major depression. Psychiatry Res 1994;55: 75-84.

21. Kostic M, Munjiza A, Pesic D, Peljto A, Novakovic I, Dobricic V, et al. A pilot study on predictors of brainstem raphe abnormality in patients with major depressive disorder. J Affect Disord 2017;209:66-70.

22. Lee HY, Tae WS, Yoon HK, Lee BT, Paik JW, Son KR, et al. Demonstration of decreased gray matter concentration in the midbrain encompassing the dorsal raphe nucleus and the limbic subcortical regions in major depressive disorder: an optimized voxel-based morphometry study. J Affect Disord 2011;133:128-136.

23. Soriano-Mas C, Hernandez-Ribas R, Pujol J, Urretavizcaya M, Deus J, Harrison BJ, et al. Cross-sectional and longitudinal assessment of structural brain alterations in melancholic depression. Biol Psychiatry 2011;69:318-325.

24. Qi H, Ning Y, Li J, Guo S, Chi M, Gao M, et al. Gray matter volume abnormalities in depressive patients with and without anxiety disorders. Medicine (Baltimore) 2014;93:e345.

25. Han KM, Kim D, Sim Y, Kang J, Kim A, Won E, et al. Alterations in the brainstem volume of patients with major depressive disorder and their relationship with antidepressant treatment. J Affect Disord 2017; 208:68-75. 
26. Bookstein FL. "Voxel-based morphometry" should not be used with imperfectly registered images. Neuroimage 2001;14:1454-1462.

27. Patenaude B, Smith SM, Kennedy DN, Jenkinson M. A Bayesian model of shape and appearance for subcortical brain segmentation. Neuroimage 2011;56:907-922.

28. Bora E, Harrison BJ, Yucel M, Pantelis C. Cognitive impairment in euthymic major depressive disorder: a meta-analysis. Psychol Med 2013; 43:2017-2026.

29. Smith R, Chen K, Baxter L, Fort C, Lane RD. Antidepressant effects of sertraline associated with volume increases in dorsolateral prefrontal cortex. J Affect Disord 2013;146:414-419.

30. Lavretsky H, Roybal DJ, Ballmaier M, Toga AW, Kumar A. Antidepressant exposure may protect against decrement in frontal gray matter volumes in geriatric depression. J Clin Psychiatry 2005;66:964-967.

31. Hamilton JP, Siemer M, Gotlib IH. Amygdala volume in major depressive disorder: a meta-analysis of magnetic resonance imaging studies. Mol Psychiatry 2008;13:993-1000.

32. American Psychiatric Association. Diagnostic and Statistical Manual of Mental Disorders. Washington, DC: American Psychiatric Press; 1994.

33. Oldfield RC. The assessment and analysis of handedness: the Edinburgh inventory. Neuropsychologia 1971;9:97-113.

34. Williams JB. A structured interview guide for the Hamilton Depression Rating Scale. Arch Gen Psychiatry 1988;45:742-747.

35. Hamilton M. Development of a rating scale for primary depressive illness. Br J Soc Clin Psychol 1967;6:278-296.

36. Park HJ, Youn T, Jeong SO, Oh MK, Kim SY, Kim EY. SENSE factors for reliable cortical thickness measurement. Neuroimage 2008;40:187196.

37. Tae WS, Kim SS, Lee KU, Nam EC, Kim KW. Validation of hippocampal volumes measured using a manual method and two automated methods (FreeSurfer and IBASPM) in chronic major depressive disorder. Neuroradiology 2008;50:569-581.

38. Winkler AM, Ridgway GR, Webster MA, Smith SM, Nichols TE. Permutation inference for the general linear model. Neuroimage 2014;92: 381-397.

39. Salimi-Khorshidi G, Smith SM, Nichols TE. Adjusting the effect of nonstationarity in cluster-based and TFCE inference. Neuroimage 2011;54:2006-2019.

40. Iglesias JE, Van Leemput K, Bhatt P, Casillas C, Dutt S, Schuff N, et al. Bayesian segmentation of brainstem structures in MRI. Neuroimage 2015;113:184-195.

41. Sled JG, Zijdenbos AP, Evans AC. A nonparametric method for automatic correction of intensity nonuniformity in MRI data. IEEE Trans Med Imaging 1998; 17:87-97.

42. Fischl B, Salat DH, Busa E, Albert M, Dieterich M, Haselgrove C, et al. Whole brain segmentation: automated labeling of neuroanatomical structures in the human brain. Neuron 2002;33:341-355.

43. Fischl B, van der Kouwe A, Destrieux C, Halgren E, Segonne F, Salat $\mathrm{DH}$, et al. Automatically parcellating the human cerebral cortex. Cereb Cortex 2004;14:11-22.

44. Benjamini Y, Hochberg Y. Controlling the false discovery rate-a practical and powerful approach to multiple testing. J R Stat Soc B 1995;57: 289-300.

45. Walter U, Prudente-Morrissey L, Herpertz SC, Benecke R, Hoeppner J. Relationship of brainstem raphe echogenicity and clinical findings in depressive states. Psychiatry Res 2007;155:67-73.

46. Bessette KL, Nave AM, Caprihan A, Stevens MC. White matter abnormalities in adolescents with major depressive disorder. Brain Imaging Behav 2014;8:531-541.

47. Budisic M, Karlovic D, Trkanjec Z, Lovrencic-Huzjan A, Vukovic V, Bosnjak J, et al. Brainstem raphe lesion in patients with major depressive disorder and in patients with suicidal ideation recorded on transcranial sonography. Eur Arch Psychiatry Clin Neurosci 2010;260:203208.

48. Han KM, Won E, Sim Y, Tae WS. Hippocampal subfield analysis in medication-naive female patients with major depressive disorder. J Affect Disord 2016;194:21-29.

49. Guo W, Liu F, Zhang Z, Liu J, Yu M, Zhang J, et al. Unidirectionally affected causal connectivity of cortico-limbic-cerebellar circuit by structural deficits in drug-naive major depressive disorder. J Affect Disord 2015;172:410-416.

50. Hornung JP. The human raphe nuclei and the serotonergic system. J Chem Neuroanat 2003;26:331-343.

51. Ahn NR, Leem YH, Kato M, Chang HK. Effects of creatine monohydrate supplementation and exercise on depression-like behaviors and raphe 5-HT neurons in mice. J Exerc Nutrition Biochem 2016;20:24-31.

52. Oquendo MA, Galfalvy H, Sullivan GM, Miller JM, Milak MM, Sublette ME, et al. Positron emission tomographic imaging of the serotonergic system and prediction of risk and lethality of future suicidal behavior. JAMA Psychiatry 2016;73:1048-1055.

53. van der Doelen RHA, Robroch B, Arnoldussen IA, Schulpen M, Homberg JR, Kozicz T. Serotonin and urocortin 1 in the dorsal raphe and Edinger-Westphal nuclei after early life stress in serotonin transporter knockout rats. Neuroscience 2017;340:345-358.

54. Young EA, Abelson JL, Cameron OG. Interaction of brain noradrenergic system and the hypothalamic-pituitary-adrenal (HPA) axis in man. Psychoneuroendocrinology 2005;30:807-814.

55. Chen VC, Shen CY, Liang SH, Li ZH, Tyan YS, Liao YT, et al. Assessment of abnormal brain structures and networks in major depressive disorder using morphometric and connectome analyses. J Affect Disord 2016;205:103-111.

56. Lai $\mathrm{CH}, \mathrm{Wu}$ YT. The gray matter alterations in major depressive disorder and panic disorder: putative differences in the pathogenesis. J Affect Disord 2015;186:1-6.

57. Peng J, Liu J, Nie B, Li Y, Shan B, Wang G, et al. Cerebral and cerebellar gray matter reduction in first-episode patients with major depressive disorder: a voxel-based morphometry study. Eur J Radiol 2011;80:395399.

58. Guillamon A, de Blas MR, Segovia S. Effects of sex steroids on the development of the locus coeruleus in the rat. Brain Res 1988;468:306310.

59. Swanson LW, Hartman BK. The central adrenergic system. An immunofluorescence study of the location of cell bodies and their efferent connections in the rat utilizing dopamine-beta-hydroxylase as a marker. J Comp Neurol 1975;163:467-505.

60. Fast CD, McGann JP. Amygdalar gating of early sensory processing through interactions with locus coeruleus. J Neurosci 2017;37:30853101 .

61. Venkatraman A, Edlow BL, Immordino-Yang MH. The brainstem in emotion: a review. Front Neuroanat 2017;11:15.

62. Chmielarz P, Kusmierczyk J, Parlato R, Schutz G, Nalepa I, Kreiner G. Inactivation of glucocorticoid receptor in noradrenergic system influences anxiety- and depressive-like behavior in mice. PLoS One 2013;8: e72632.

63. Klimek V, Stockmeier C, Overholser J, Meltzer HY, Kalka S, Dilley G, et al. Reduced levels of norepinephrine transporters in the locus coeruleus in major depression. J Neurosci 1997;17:8451-8458.

64. Shibata E, Sasaki M, Tohyama K, Otsuka K, Sakai A. Reduced signal of locus ceruleus in depression in quantitative neuromelanin magnetic resonance imaging. Neuroreport 2007;18:415-418.

65. Barde S, Ruegg J, Prud'homme J, Ekstrom TJ, Palkovits M, Turecki G, et al. Alterations in the neuropeptide galanin system in major depressive disorder involve levels of transcripts, methylation, and peptide. Proc Natl Acad Sci U S A 2016;113:E8472-E8481.

66. Chandley MJ, Szebeni A, Szebeni K, Crawford JD, Stockmeier CA, Turecki $\mathrm{G}$, et al. Elevated gene expression of glutamate receptors in noradrenergic neurons from the locus coeruleus in major depression. Int J Neuropsychopharmacol 2014;17:1569-1578.

67. Ghosal S, Bundzikova-Osacka J, Dolgas CM, Myers B, Herman JP. Glucocorticoid receptors in the nucleus of the solitary tract (NTS) de- 
crease endocrine and behavioral stress responses. Psychoneuroendocrinology 2014;45:142-153.

68. Saha S, Gamboa-Esteves FO, Batten TF. Differential distribution of 5-HT 1A and 5-HT 1B-like immunoreactivities in rat central nucleus of the amygdala neurones projecting to the caudal dorsomedial medulla oblongata. Brain Res 2010;1330:20-30.

69. Sofroniew MV, Vinters HV. Astrocytes: biology and pathology. Acta Neuropathol 2010;119:7-35.

70. Kimelberg HK. Functions of mature mammalian astrocytes: a current view. Neuroscientist 2010;16:79-106.

71. Czeh B, Di Benedetto B. Antidepressants act directly on astrocytes: evidences and functional consequences. Eur Neuropsychopharmacol 2013;23:171-185.

72. Bowley MP, Drevets WC, Ongur D, Price JL. Low glial numbers in the amygdala in major depressive disorder. Biol Psychiatry 2002;52:404412.

73. Rajkowska G, Miguel-Hidalgo JJ. Gliogenesis and glial pathology in depression. CNS Neurol Disord Drug Targets 2007;6:219-233.

74. Harrison PJ. The neuropathology of primary mood disorder. Brain 2002;125:1428-1449.

75. Riecher-Rossler A. Sex and gender differences in mental disorders. Lancet Psychiatry 2017;4:8-9.

76. Kuehner C. Why is depression more common among women than among men? Lancet Psychiatry 2017;4:146-158.

77. Kessler RC. Epidemiology of women and depression. J Affect Disord 2003; 74:5-13.

78. Kessler RC, Berglund P, Demler O, Jin R, Koretz D, Merikangas KR, et al. The epidemiology of major depressive disorder: results from the National Comorbidity Survey Replication (NCS-R). JAMA 2003;289: 3095-3105.
79. Kessler RC, Berglund P, Demler O, Jin R, Merikangas KR, Walters EE. Lifetime prevalence and age-of-onset distributions of DSM-IV disorders in the National Comorbidity Survey Replication. Arch Gen Psychiatry 2005;62:593-602.

80. Kuehner C. Gender differences in unipolar depression: an update of epidemiological findings and possible explanations. Acta Psychiatr Scand 2003;108:163-174.

81. de Graaf R, ten Have M, van Gool C, van Dorsselaer S. Prevalence of mental disorders and trends from 1996 to 2009. Results from the Netherlands Mental Health Survey and Incidence Study-2. Soc Psychiatry Psychiatr Epidemiol 2012;47:203-213.

82. Wittchen HU, Jacobi F, Rehm J, Gustavsson A, Svensson M, Jonsson B, et al. The size and burden of mental disorders and other disorders of the brain in Europe 2010. Eur Neuropsychopharmacol 2011;21:655-679.

83. Jacobi F, Hofler M, Siegert J, Mack S, Gerschler A, Scholl L, et al. Twelvemonth prevalence, comorbidity and correlates of mental disorders in Germany: the Mental Health Module of the German Health Interview and Examination Survey for Adults (DEGS1-MH). Int J Methods Psychiatr Res 2014;23:304-319.

84. Lim GY, Tam WW, Lu Y, Ho CS, Zhang MW, Ho RC. Prevalence of Depression in the Community from 30 Countries between 1994 and 2014. Sci Rep 2018;8:2861.

85. Byers AL, Yaffe K, Covinsky KE, Friedman MB, Bruce ML. High occurrence of mood and anxiety disorders among older adults: the National Comorbidity Survey Replication. Arch Gen Psychiatry 2010;67: 489-496.

86. Luppa M, Sikorski C, Luck T, Ehreke L, Konnopka A, Wiese B, et al. Age- and gender-specific prevalence of depression in latest-life--systematic review and meta-analysis. J Affect Disord 2012;136:212-221. 